\title{
Stand der Reform der ärztlichen Gebührenordnungen aus Sicht des BDDH e. V.
}

Zur Abrechnung mit der Kassenärztlichen Vereinigung wird bei sozialversicherten Patienten im ambulanten Bereich der EBM (einheitlicher Bewertungsmaßstab) verwendet, zur Abrechnung bei Privatpatienten, Selbstzahlern und im Rahmen der Unfallversicherung/ Berufsgenossenschaften hingegen die GOÄ (Gebührenordnung für Ärzte). Hinter beiden Abrechnungssystemen steht eine sehr unterschiedliche Philosophie: Einmal die Versorgung Sozialversicherter mit den notwendigen medizinischen Leistungen, zum anderen die Honorierung des Arztes für seine freiberufliche Tätigkeit. Dies ist der Grund, warum ähnliche Leistungen in beiden Systemen unterschiedlich bezahlt werden und auch andere Partner an einer Modernisierung und Reform dieser Regelwerke beteiligt sind. Sowohl bei dem EBM als auch bei der GOÄ ist eine Reform seit Jahren überfällig aber absolut erforderlich, um einerseits neu etablierte medizinische Leistungen und auch neue hämostaseologische Untersuchungen abbilden zu können und auch die Honorierung einzelner Leistungen an die aktuelle Kostensituation (Personalaufwand, Reagenzien, Analyzer, etc.) anzupassen.

Die große Koalition hat zu Beginn ihrer Zusammenarbeit in dieser Legislaturperiode eine wissenschaftliche Kommission eingesetzt, da die beiden diskutierten Modelle, insbesondere Beibehaltung von zwei großen Versicherungssystemen (CDU) vs. Bürgerversicherung (SPD) in der Koalitionsverhandlung nicht vereinbar waren. Ein Resultat war bisher das Terminservice- und Versorgungsgesetz (TSG), mit dem Unterschiede bei Privat- und Kassenversicherten, insbesondere beim zügigen Zugang zu Fachärzten, aufgehoben werden soll. Es bleibt abzuwarten, ob hier nicht ein Bürokratiemonster geschaffen wird, welches die Patienten wenig nutzen. Im Weiteren ist es wenig wahrscheinlich, dass sich in der verbleibenden Regierungszeit diesbezüglich noch etwas bewegt. Was bisher erarbeitetet wurde, stellen wir Ihnen unten dar. Was daraus in welchem Umfang realisiert werden wird, insbesondere wenn sich die politischen Konstellationen bzw. die Regierungsverantwortung ändern sollten, ist derzeit nicht abzusehen.

\section{EBM - Einheitlicher Bewertungsmaßstab}

Der letzte Kontakt des BDDH mit der Kassenärztlichen Bundesvereinigung KBV bestand am 17. Mai 2019 im Rahmen der Konzertierten Aktion der KBV mit den Berufsverbänden. Aktuelle Veränderungen am EBM ergeben sich vor allem durch das TSG. Hier gibt es dann eine extrabudgetäre Vergütung von Leistungen im Behandlungsfall, wenn diese aufgrund einer Terminvermittlung erfolgen. Ab September soll es auch zu Zuschlägen von bis zu 50\% kommen. Auch wenn die Hausärzte

direkt dringende Termine bei Fachärzten vermitteln, erhalten diese ihre Vergütung extrabudgetär. Der Hausarzt erhält hierfür $10 €$. Neben Personaleinstellungen bei den KVen muss für dieses Gesetz auch eine weitere Telematikinfrastruktur aufgebaut werden; die praktische Umsetzung gestaltet sich jedoch schwierig, der gesetzte Zeitrahmen wird flächendeckend nicht eingehalten werden können. In wieweit wir Hämostaseologen im ambulanten Bereich von den Neuregelungen durch das TSG profitieren können, bleibt fraglich und von der Facharztgruppe abhängig.

Die eigentliche Weiterentwicklung des EBM dauert seit 2012 an. Verbesserungen hinsichtlich der Entwicklung und Erweiterung der Leistungsbeschreibung, der Sicherstellung einer flächendeckenden Versorgung, der Honorargerechtigkeit zwischen und innerhalb der Arztgruppen, der wirtschaftlichen und bedarfsgerechten Leistungserbringung unterliegen der Ausgabenneutralität. Ein neuer EBM darf also in der Summe nicht mehr kosten.

Beratungen zwischen KBV und GKV-Spitzenverband werden seit 2017 wieder durchgeführt, diese sollen im Sommer 2019 abgeschlossen sein. Dabei wird es aber wohl eher zu einer Art „kleiner Reform“ mit Fokussierung auf aktuell notwendige Änderungen kommen. Es soll keine signifikante Umverteilung zwischen den Arztgruppen entstehen. Die Einführung neuer Leistungen im EBM soll daher vor dem Hintergrund einer Punktsummenneutralität zurückgestellt werden. Dieses hatte der BDDH in Zusammenarbeit mit der GTH schon beim Versuch der Einführung einer Beratungsziffer für komplexe Gerinnungsstörungen erfahren. Diese könnte nur kostenneutral eingeführt werden. Es gibt aber keine Stelle, wo dann hierfür gekürzt werden könnte.

Der kalkulatorische Arztlohn soll angepasst werden und entspricht dann dem eines leitenden Oberarztes. Dies wird aber mit den GKV-Spitzenverbänden erst nach Abschluss der Verhandlungen zu Arztleistungszeiten und medizinischer Plausibilisierung besprochen.

Die KBV versucht als weiteres Vorgehen eine Plausibilisierung der vorläufig rechnerischen Ergebnisse gemeinsam mit dem GKV-Spitzenverband und erstellt zurzeit eine Simulationsberechnung zu den Auswirkungen einer EBM-Reform. Sofern kein Konsens mit dem GKV-Spitzenverband möglich ist, wird die KBV einen eigenen EBM-Entwurf vorlegen.

\section{GOÄ - Gebührenordnung für Ärzte}

Die Bundesärztekammer (BÄK) hat vom Bundesministerium für Gesundheit (BfG) zusammen mit der Vereinigung der privaten Krankenversicherer den Auftrag erhalten, einen

(c) 2019 Georg Thieme Verlag KG Stuttgart · New York
DOI https://doi.org/

$10.1055 / \mathrm{s}-0039-1693496$. ISSN 0720-9355. 
Vorschlag für eine GOÄ-Reform zu erarbeiten. Dabei wurden von der BÄK die Berufsverbände (130 und später noch einmal 65) über Jahre eingebunden, um in zahlreichen Veranstaltungen die in einzelnen Gebührenordnungsziffern enthaltenen Leistungen exakt zu definieren und dann anhand der Berechnung von ärztlicher, personeller, technischer Leistung unter Addition der Gemeinkosten zu einer Bewertung zu bringen. Die bisher verwendeten Steigerungsätze werden dann mit der neu gefassten GOÄ (GOÄneu) entfallen. Bisher steht die Bewertung für über 6000 Ziffer, was einem Abdeckungsgrad von $80 \%$ entspricht. Auch die Transkodierung ist mit einem Abdeckungsgrad von über 65\% fortgeschritten. Diese wird gebraucht, um alte und neue GOÄ vergleichbar zu halten und abschätzen zu können, in welcher Konstellation der Nachfrage von GOÄ-Leistung die GOÄneu zu einer Vergütungssteigerung führt. Insgesamt sollte es nämlich nicht zu einer Steigerung von mehr als 6,4\% kommen, wenn man für die gleiche Leistungsmenge (z. B. alle GOÄ-Leistungen des Jahres 2017) nach GOÄneu statt GOÄalt abrechnet. 6,4\% entspricht dabei einem alten Stand und kann daher inzwischen etwas höher ausfallen.

Am 6. Juni 2019 hat in Berlin die „Auftaktveranstaltung zur Finalisierung der Bewertungen der GOÄneu“ stattgefunden. Hier waren die medizinischen Fachgesellschaften und Berufsverbände, unter anderem auch der BDDH e.V., von der BÄK geladen. Weitere Veranstaltungen zu einzelnen Kapiteln werden reichlich folgen. Jedoch ist das für den Hämostaseologen interessante Kapitel „Laborleistungen“ hiervon erst einmal ausgenommen. Was hier in Zukunft passieren wird, wird vom BDDH weiter aufmerksam verfolgt. Gerade die Bewertungen für Gerinnungsanalysen scheinen aber nicht verschlechtert zu werden. An den Definitionen der Laborziffern für Gerinnungsanalytik war der BDDH beteiligt. Bei einer GOÄneu wären dann alle nach heutigem Stand sinnvollen Analysen abrechenbar, ohne dass Analogziffern verwendet werden müssten.

Ziel dieser Veranstaltungen ist es, der jetzt vorliegen Version 2.0 noch einen Feinschliff hinsichtlich Behebung offensichtlicher Bewertungsfehler und Implausibilitäten zu geben. Im späteren Verlauf sind die Bewertungen dann so abzugleichen, dass insgesamt der oben genannte Steigerungsrahmen erhalten bleibt und dies mit der PKV verhandelt werden kann.

Falls PKV und BÄK einen gemeinsamen Vorschlag zur GOÄneu finden, soll dieser dem MfG vorgelegt werden. Die wissenschaftliche Kommission erstellt zudem ein eigenes Gutachten. Letztendlich muss der Bundesrat die GOÄneu beschließen. Dessen Mitglieder, die Bundesländer, haben als Beihilfeträger auch noch einmal gesonderte Interessen.

\section{Fazit}

In eine Reform von EBM und GOÄ ist bereits über Jahre von vielen Seiten viel Arbeit eingeflossen. Ob sich dies am Ende lohnen oder dann von der Politik überholt wird, zeigt vielleicht die nächste Legislaturperiode. Daher gibt es keine klare Entwicklung in den Gebührenordnungen, auf die wir uns als Hämostaseologen einstellen können.

Für den Vorstand des Berufsverbandes der Deutschen Hämostaseologen e.V. (BDDH)

Dr. med. Günther Kappert

PD Dr. med. Christoph Sucker

\section{Aktuelle Informationen zur Abstimmung am 28.06.2019 zum Gesetz für mehr Sicherheit in der Arzneimittelver- sorgung (GSAV) im Bundesrat}

In der Sitzung am 28.06.2019 im Bundesrat hat dieser dem GSAV zugestimmt. Aber es gab zusätzlich zwei ENTSCHLIESSUNGEN, auch auf Empfehlung des Gesundheitsausschusses des Bundestages. Die Entschließung 254/19b (Beschluss) betrifft die "Hämophilie" - insbesondere die Änderung des Vertriebsweges (§ 47.2 AMG). Hierin wird die Änderung als $\mathrm{zu}$ “drastischer Eingriff" bezeichnet und “eine Schwächung der Zentrumsversorgung" befürchtet!

Die Begründung fasst auch erstmal ALLE Argumente der gemeinsamen konzertierten Aktionen zusammen. Auch werden "die innovativen Versorgungsverträge zwischen Krankenkassen und Hämophiliezentren" hervorgehoben und als Argument gegen die Änderung des Vertriebsweges angeführt.

"Solche Verträge gibt es mittlerweile flächendeckend mit den Ersatzkassen (vdek-Vertrag) sowie mit einigen regionalen AOKen und Betriebskrankenkassen. Bei diesen Verträgen wird auch der wirtschaftliche Einsatz der Arzneimittel zur Vertragsgrundlage gemacht. Das mit der
Regelung beabsichtigte Ziel, den „aktuellen Entwicklungen in der spezifischen Therapie von Gerinnungsstörungen bei Hämophilie Rechnung zu tragen“, sollte auch mit weniger drastischen Eingriffen in die Versorgung dieser sensiblen Patientengruppe erreichbar sein." Explizit werden die Krankenkassen aufgerufen, bereits jetzt Rabattverträge nach $\S$ 130a SGB V oder $\S 130$ c SGB V zu schließen, um mehr Preistransparenz zu gewährleisten, was ja das eigentliche Anliegen des BMG war und ist.

Unterstützt wird die Entschließung u.a. durch die Bundesärztekammer und die Arzneimittelkommission der deutschen Ärzteschaft, die die Positionen u.a. des BDDH, der GTH und der DGTI aufgenommen haben.

Im Übrigen wurde über die konzertierte Aktion des BDDH und der GTH Kontakt zum Bundeskanzleramt bereits hergestellt, um nochmals die gemeinsame Position, die im Wesentlichen der Entschließung 254/19b (Beschluss) entspricht, erneut darzulegen. 


\section{Rechtsinfo "Entschließung”}

Der Bundesrat hat neben Bundestag und Bundesregierung zudem ein Initiativrecht in der Gesetzgebung (Artikel 76 Abs. $1 \mathrm{GG}$ ). Die vom Bundesrat beschlossenen Gesetzentwürfe werden zunächst der Bundesregierung zugeleitet. Sie kann innerhalb von sechs Wochen - in besonderen Fällen innerhalb von drei oder neun Wochen - eine Stellungnahme dazu abgeben. Danach ist der Gesetzentwurf an den Bundestag weiterzuleiten.

Als eine politische Ergänzung des Initiativrechts wird auch das parlamentarische Mittel der Entschließung eingesetzt. Darunter versteht man Ersuchen, die in der Regel an die Bundesregierung gerichtet sind, um auf Probleme aufmerksam zu machen, die Auffassung des Bundesrates zu einem bestimmten Thema darzulegen oder Gesetzgebungsverfahren durch die Bundesregierung anzustoßen. Entschließungen sind rechtlich jedoch nicht verbindlich.

\section{Weiterer Verlauf}

Der Staatssekretär im Bundesministerium für Gesundheit, Dr. Thomas Steffen, hat in seiner Rede vor dem Plenum des Bundesrates angekündigt und protokollarisch niedergelegt, dass die Bundesregierung spätestens sechs Monate nach Inkrafttreten des Gesetzes einen Erfahrungsbericht zum Bereich Hämophilieversorgung vorlegen wird und geht damit bereits auf die Entschließung 254/19b zum Thema Hämophilie ein.

Anders als beim 1. Durchgang im Bundesrat, wird die Bundesregierung nun keine erneute Gegenäußerung oder ähnliches zur Entschließung formulieren. Sie wird sich damit auch nicht erneut mit dem Thema im Rahmen dieses Gesetzes auseinandersetzen.
Damit ist das GSAV angenommen. Nach anschließender Vorlage beim Bundespräsidenten wird das GSAV in unveränderter Form im Bundesanzeiger gedruckt werden und verbindlich rechtsgültig werden.

\section{Fazit}

Die Änderung des Vertriebsweges nach § 47.2 AMG ist im GSAV mit einer einjährigen Übergangsfrist beschlossen worden. Es ist sehr, sehr positiv zu bewerten, dass Alle aktiv Beteiligten im Bereich der Hämophilieversorgung außerhalb des BMG ein großes Engagement, ein hohes Problembewußtsein und einen offenkundigen Lösungswillen im Sinne der "guten" Patientenversorgung dokumentiert haben.

https://www.bundesrat.de/SharedDocs/TO/979/to-node. html?cms_topNr=6 (überprüft am 29.06.2019)

- Bundesrat Drucksache 254/19 (Beschluss) 28.06.19

- Bundesrat Drucksache 254/19 Erläuterung, 979. BR, 28.06.19

Autor für den BDDH:

Priv.-Doz. Dr. med. Jürgen Koscielny

Stellvertretender Vorsitzender des BDDH

Charité - Universitätsmedizin Berlin, Campus Charité Mitte (CCM)

Charitéplatz 1, Durchgang Luisenstr. 13

D-10117 Berlin (Germany)

e-mail: juergen.koscielny@charite.de 